University of Massachusetts Amherst

ScholarWorks@UMass Amherst

1987

\title{
Analytical Information from Doublet Peaks in Flow Injection Analysis Part I. Basic Equation and Applications to Flow Injection Titrations
}

Julian Tyson

University of Massachusetts Amherst

Follow this and additional works at: https://scholarworks.umass.edu/chem_faculty_pubs

Part of the Chemistry Commons

\section{Recommended Citation}

Tyson, Julian, "Analytical Information from Doublet Peaks in Flow Injection Analysis Part I. Basic Equation and Applications to Flow Injection Titrations" (1987). Analyst. 1382.

Retrieved from https://scholarworks.umass.edu/chem_faculty_pubs/1382 


\title{
Analytical Information from Doublet Peaks in Flow Injection Analysis Part I. Basic Equation and Applications to Flow Injection Titrations*
}

\author{
Julian F. Tyson \\ Department of Chemistry, University of Technology, Loughborough, Leicestershire LE11 3TU, UK
}

\begin{abstract}
An equation relating the peak separation $\left(\Delta t_{\text {eq }}\right)$, between the doublet peak obtained when the reaction product of a flow injection titration is monitored, to all the basic operational variables was derived on the basis of the well stirred mixing chamber model. These variables consisted of the flow-rate, the volume injected, the volume of the mixing chamber and the concentrations of the reagent and the injection sample. The equation was tested for the predicted variation of $\Delta t_{\mathrm{eq}}$ with these variables for a system containing real mixing chambers but using slug injection. The reactants were copper(II) and EDTA and the product was monitored with a UV detector. Good agreement was obtained. The use of a gradient tube was demonstrated to give a good calibration for $\mathrm{OH}^{-}$over the range $5 \times 10^{-5}-5 \mathrm{~m}$. A comparison of the doublet peak method with potentiometric methods was made.
\end{abstract}

Keywords: Flow injection analysis; doublet peaks; flow injection titrations; copper(II); EDTA

The most commonly used quantitative parameter in flow injection analysis (FIA) is peak height. Little use has been made of peak area, although its usefulness in some situations (such as in flow injection atomic absorption spectrometry) has been demonstrated. ${ }^{1}$ The use of peak width as a quantitative parameter in FIA was demonstrated by Růžička, et al. ${ }^{2}$ who derived equations based on the tanks-in-series model reduced to the situation for just one tank. The approach adopted followed that of earlier workers in the field of continuous flow titrations $^{3-5}$ (so called because conditions are adjusted so that there is a point on both the rise and fall graphs at which the concentrations of the sample and the reagent are in their stoicheiometric equivalences) in that the concentration of one of the reactants was followed as a function of time. Possibly the most interesting feature of this mode of monitoring in FIA, namely the considerable extension of the useful working range (as the peak width is related to the logarithm of the sample concentration), was stressed only later. ${ }^{6}$ This logarithmic relationship arises from the generation of the exponential concentration gradients of the sample and the reagent. This can be conveniently achieved by the use of a well stirred mixing chamber. Ramsing et al. ${ }^{7}$ showed later that a real mixing chamber was unnecessary, so acceptable results could be obtained much faster from using the injector, a short connecting line and a detector to produce the mixing in the system. The use of this type of manifold with a variety of detectors has recently been described by Rhee and Dasgupta. 8,9 The application of peak width monitoring in systems where only physical dispersion occurs for extending the normal working range has been clearly demonstrated for systems both with ${ }^{10}$ and without ${ }^{11}$ well stirred mixing chambers.

When the product of a chemical reaction is monitored under conditions at which equivalence is obtained on the rise and fall graphs, two peaks will be observed as the injected material (the sample) will be in excess in the profile centre. ${ }^{2}$ Equations relating the time interval between the doublet peaks to the injected concentration have been derived without approximations for both the single-line and merging-stream manifold. ${ }^{13}$ This paper also showed that the restrictions of maintaining (a) the injected sample volume less than the mixing tank volume and (b) the injected sample concentration greater than the reagent carrier stream concentration could be relaxed.

\footnotetext{
* Presented at SAC 86, the 7th SAC International Conference on
} Analytical Chemistry, Bristol, UK, 20-26 July, 1986.
Further, as the peaks occur at the equivalence points, the location of the appropriate measurement level is much simpler than when a reactant profile is followed. The method was illustrated by the monitoring of the doublet peaks of copper EDTA produced by the injection of $500 \mu \mathrm{l}$ of copper solutions covering the range $1.6 \times 10^{-6}-0.16 \mathrm{M}$ into $10^{-4} \mathrm{M}$ of EDTA, with a coiled tubular reactor of approximately $400 \mu$ l volume, which behaved as a well stirred mixing chamber of about $100 \mu \mathrm{l}$.

In this paper, the equation previously derived ${ }^{13}$ is extended to account for the effect of the volume injected. The validity of this equation is investigated for all of the experimental parameters including the volume injected, the flow-rate and the mixing chamber volume. Other gradient-forming devices are examined and the use of a gradient tube illustrated for the determination of the hydroxide ion.

\section{Basic Equation}

The underlying model assumes the plug flow (at a flow-rate $Q$ $\mu l \mathrm{~s}^{-1}$ ) of the injected sample (volume $V_{\mathrm{i}} \mu \mathrm{l}$, concentration $C_{\mathrm{o}}^{\mathrm{S}} \mathrm{M}$ ) through a well stirred mixing chamber (volume $V \mu \mathrm{l}$ ) containing the reagent (concentration $C_{\mathrm{o}}^{\mathrm{R}} \mathrm{M}$ ). The time interval $\Delta t_{\text {eq }} \mathrm{s}$ ) between the doublet peaks is given by ${ }^{13}$

$$
\Delta t_{\mathrm{eq}}=(V / Q) \ln C_{\mathrm{o}}^{\mathrm{S}}-(V / Q) \ln C_{\mathrm{o}}^{\mathrm{R}}(D-1) \quad \ldots
$$

where $D$ is the dispersion coefficient of the injected sample material defined as the ratio $C_{\mathrm{o}}^{\mathrm{S}} / C_{\mathrm{p}}^{\mathrm{S}}$ where $C_{\mathrm{p}}^{\mathrm{s}}$ is the concentration of the sample that would be obtained at the peak maximum of the dispersed sample zone in the absence of a chemical reaction. The dispersion coefficient is given by ${ }^{13}$

$$
D=\left[1-\exp \left(-V_{\mathrm{i}} / V\right)\right]^{-1}
$$

Substitution of equation (2) into equation (1) and rearranging gives

$\Delta t_{\mathrm{eq}}=(V / Q) \ln C_{\mathrm{o}}^{\mathrm{S}}-(V / Q) \ln C_{\mathrm{o}}^{\mathrm{R}}+(V / Q) \ln \left[\exp \left(V_{\mathrm{i}} / V\right)-1\right](3)$ Equation (3) can be simplified further to a number of versions such as

$$
\Delta t_{\mathrm{eq}}=(V / Q) \ln C_{\mathrm{o}}^{\mathrm{s}}\left[\exp \left(V_{\mathrm{i}} / V\right)-1\right] / C_{\mathrm{o}}^{\mathrm{R}}
$$

Equation (4) shows the expected variations of $\Delta t_{\mathrm{eq}}$ with the experimental variables, all of which are readily envisaged apart from the effect of changing the volume injected. It can be shown [for example by differentiation of equation (4)] that the function relating $\Delta t_{\mathrm{eq}}$ to $V$ only has a maximum value when $C_{\mathrm{o}}^{\mathrm{S}} / C_{\mathrm{o}}^{\mathrm{R}}>1$.

By setting $\Delta t_{\mathrm{eq}}$ equal to zero, equation (4) can be used firstly to calculate the limit of detection for any given experimental 
arrangement. Hence the minimum concentration $C_{\min }^{\mathrm{s}}$ is given by

$$
C_{\text {min. }}^{\mathrm{s}}=C_{\mathrm{o}}^{\mathrm{R} /\left[\exp \left(V_{\mathrm{i}} / V\right)-1\right]} \ldots
$$

Equation (5) shows that, for given volumes injected and volumes of the mixing chamber, the limit of detection is directly proportional to the concentration of the reagent in the carrier stream. Further, $C_{\text {min. }}^{\mathrm{S}}$ will only be less than $C_{\mathrm{o}}^{\mathrm{R}}$ if $\left[\exp \left(V_{\mathrm{i}} / V\right)-1\right]>1$, and this gives an upper limiting ratio for $V_{\mathrm{i}} / V$ of 0.693 . Secondly, rearrangement of equation (5) gives

$$
V_{\mathrm{i}} / V=\ln \left[\left(C_{\mathrm{o}}^{\mathrm{S}} / C_{\mathrm{o}}^{\mathrm{R}}\right)+1\right]
$$

giving the limiting value of the ratio of $V_{\mathrm{i}} / V$ for known values of $C_{\mathrm{o}}^{\delta}$ and $C_{\mathrm{o}}^{\mathrm{R}}$, hence enabling suitable choices of values of $V_{\mathrm{i}}$ and $V$ to be made to allow a particular detection limit to be reached.

\section{Experimental}

\section{Apparatus}

The flow injection system (see Fig. 1) consisted of an Ismatec Model MS-4REGLO peristaltic pump, an Altex Type 201-25 eight-port slider injection valve and a Pye Unicam Model PU 4020 UV HPLC detector. Connecting tubing was $0.71 \mathrm{~mm}$ i.d. PTFE tubing and various end fittings and connectors (Tecator, Altex) were used. The output from the detector was monitored either by a Tekman Model TE 200 chart recorder or a Spectra-Physics Type SP 4270 computing integrator. The time base of the integrator was set to monitor retention times to the nearest $0.1 \mathrm{~s}$. The mixing chambers were of sectional

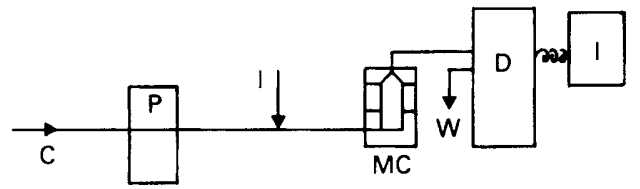

Fig. 1. Schematic diagram of flow injection analysis system. C, carrier stream; I, injection valve; $\mathrm{MC}$, three-section mixing chamber (replaced by the gradient tube for the experiments involving $\mathrm{OH}^{-}$ determination); D, HPLC detector; W, waste; and I, computing integrator construction similar to that described in reference 2 . The base part contained a cylindrical cavity, approximately $10 \mathrm{~mm}$ in diameter and $10 \mathrm{~mm}$ deep, which housed a magnetic follower. The inlet was machined so as to be tangential to the base of the cylinder in a direction to induce flow in the chamber in the opposite sense to that due to rotation of the magnetic follower. The upper section was conical with base $10 \mathrm{~mm}$ and height approximately $4 \mathrm{~mm}$, with the outlet machined vertically at the apex of the cone. Three middle slices of $10 \mathrm{~mm}$ circular bore and heights approximately 5,10 and $20 \mathrm{~mm}$ could be inserted to produce tanks with a variety of volumes. Joints between the sections were sealed with gaskets made from PTFE plumbers tape. The gradient tube from a Tecator FIAstar Model 5001 system consisting of a straight Perspex tube approximately $50 \mathrm{~mm}$ long and $2 \mathrm{~mm}$ i.d. was used in the calibration for the $\mathrm{OH}^{-}$experiment.

\section{Reagents}

Solutions of approximately $0.01 \mathrm{~m}$ EDTA and $0.01 \mathrm{M} \mathrm{Cu}^{\mathrm{II}}$ were prepared by the dissolution of the appropriate amounts of the AnalaR grade disodium salt and sulphate, respectively, in singly distilled water. The EDTA was standardised against a standard zinc solution in the presence of hexammine buffer using xylenol orange as the indicator. The copper was standardised against the EDTA under the same conditions.

The sodium hydroxide solutions were produced by serial dilution of a $5 \mathrm{~m}$ bench reagent stock, and the reagent stream was $5 \times 10^{-4} \mathrm{M} \mathrm{HCl}$ with a few drops of bromothymol blue indicator solution per $100 \mathrm{ml}$.

\section{Preliminary Experiments}

A range of copper concentrations were injected $(482 \mu \mathrm{l})$ into approximately $10^{-4} \mathrm{M}$ EDTA carrier and the product profiles monitored at $280 \mathrm{~nm}$. Graphs of $\Delta t_{\mathrm{eq}}$ versus $\ln C_{\mathrm{o}}^{\mathrm{s}}$ were constructed for a variety of gradient-forming devices including straight and coiled tubes (different radii of coiling), the gradient tube and the well stirred mixing chamber. The slopes and correlation coefficients were compared.

\begin{tabular}{|c|c|c|c|c|c|c|c|c|c|c|}
\hline Run No. & $V_{\mathrm{i}} / \mu \mathrm{l}$ & $V / \mu l$ & $Q / \mu l s^{-1}$ & $C_{\mathrm{o}}^{\mathrm{S}} / \mathrm{M}$ & $C_{\mathrm{o}}^{\mathrm{R}} / \mathrm{M}$ & \multicolumn{3}{|c|}{ Measured $\Delta t_{\mathrm{eq}} / \mathrm{s}$} & $\begin{array}{l}\text { Mean } \\
\Delta t_{\text {eq }} / \mathrm{s}\end{array}$ & $\begin{array}{c}\text { Calculated } \\
\Delta t_{\mathrm{eq}} / \mathrm{s}\end{array}$ \\
\hline 1 & 1410 & 378 & 31.5 & $1.012 \times 10^{-5}$ & $1.003 \times 10^{-4}$ & 17.3, & 17.3 & 16.6 & 17.1 & 16.9 \\
\hline 2 & 1410 & 378 & 31.4 & $1.012 \times 10^{-4}$ & $1.003 \times 10^{-4}$ & 46.3 , & 46.6 & 46.5 & 46.5 & 44.7 \\
\hline 3 & 1410 & 378 & 31.2 & $1.012 \times 10^{-3}$ & $1.003 \times 10^{-4}$ & 72.4 & 72.5 & 72.5 & 72.5 & 72.9 \\
\hline 4 & 1410 & 378 & 31.2 & $1.012 \times 10^{-2}$ & $1.003 \times 10^{-4}$ & 97.9 & 98.4 & 97.9 & 98.1 & 100.8 \\
\hline 5 & 913 & 378 & 23.2 & $1.003 \times 10^{-5}$ & $1.012 \times 10^{-5}$ & 29.3 & 32.1 & 29.8 & 30.4 & 37.7 \\
\hline 6 & 913 & 378 & 23.3 & $1.003 \times 10^{-4}$ & $1.012 \times 10^{-5}$ & 73.5 & 73.9 & 73.9 & 73.8 & 74.9 \\
\hline 7 & 913 & 378 & 23.3 & $1.003 \times 10^{-3}$ & $1.012 \times 10^{-5}$ & 108.9, & 106.6, & 109.2 & 108.2 & 112.2 \\
\hline 8 & 98 & 378 & 26.9 & $1.003 \times 10^{-3}$ & $1.012 \times 10^{-4}$ & 15.6 & 14.8, & 15.6 & 15.3 & 15.1 \\
\hline 9 & 198 & 378 & 27.6 & $1.003 \times 10^{-3}$ & $1.012 \times 10^{-4}$ & 26.4 & 26.9 & 26.7 & 26.7 & 26.3 \\
\hline 10 & 482 & 378 & 27.1 & $1.003 \times 10^{-3}$ & $1.012 \times 10^{-4}$ & 45.8 & 46.0 & 43.7 & 45.2 & 45.2 \\
\hline 11 & 913 & 378 & 27.8 & $1.003 \times 10^{-3}$ & $1.012 \times 10^{-4}$ & 63.4 & 64.6 & 64.6 & 64.2 & 62.7 \\
\hline 12 & 1410 & 378 & 26.9 & $1.003 \times 10^{-3}$ & $1.012 \times 10^{-4}$ & 84.3 & 85.2 & 85.9 & 85.1 & 84.3 \\
\hline 13 & 1410 & 781 & 26.4 & $1.003 \times 10^{-3}$ & $1.012 \times 10^{-4}$ & 115.7, & 115.7 & 115.2 & 115.5 & 120.5 \\
\hline 14 & 913 & 781 & 26.0 & $1.003 \times 10^{-3}$ & $1.012 \times 10^{-4}$ & 89.0 & 89.8 & 90.5 & 89.8 & 92.8 \\
\hline 15 & 482 & 781 & 25.6 & $1.003 \times 10^{-3}$ & $1.012 \times 10^{-4}$ & 63.4 & 63.4 & 66.9 & 64.6 & 65.1 \\
\hline 16 & 198 & 781 & 26.4 & $1.003 \times 10^{-3}$ & $1.012 \times 10^{-4}$ & 30.2 & 30.3 & 30.2 & 30.2 & 31.2 \\
\hline 17 & 482 & 1167 & 25.2 & $1.003 \times 10^{-3}$ & $1.012 \times 10^{-4}$ & 71.2 & 72.7 & 72.7 & 72.2 & 75.2 \\
\hline 18 & 913 & 1167 & 25.7 & $1.003 \times 10^{-3}$ & $1.012 \times 10^{-4}$ & 108.7, & 108.2, & 109.0 & 108.6 & 111.9 \\
\hline 19 & 1410 & 1167 & 25.7 & $1.003 \times 10^{-3}$ & $1.012 \times 10^{-4}$ & 140.2, & 139.5 & 139.9 & 139.9 & 142.9 \\
\hline 20 & 1892 & 1167 & 25.6 & $1.003 \times 10^{-3}$ & $1.012 \times 10^{-4}$ & 164.9, & 166.8, & 165.8 & 165.8 & 168.4 \\
\hline 21 & 1892 & 1941 & 25.4 & $1.003 \times 10^{-3}$ & $1.012 \times 10^{-4}$ & 204.2 , & 207.6 & 206.7 & 206.2 & 213.6 \\
\hline 22 & 913 & 1941 & 25.4 & $1.003 \times 10^{-3}$ & $1.012 \times 10^{-4}$ & 131.0, & 134.1, & 133.9 & 133.0 & 136.3 \\
\hline 23 & 482 & 781 & 31.2 & $1.003 \times 10^{-3}$ & $1.012 \times 10^{-4}$ & 53.7 & 51.8 & 52.3 & 52.6 & 53.5 \\
\hline 24 & 482 & 781 & 25.3 & $1.003 \times 10^{-3}$ & $1.012 \times 10^{-4}$ & 65.7 & 64.8 , & 65.3 & 65.3 & 65.9 \\
\hline 25 & 482 & 781 & 18.5 & $1.003 \times 10^{-3}$ & $1.012 \times 10^{-4}$ & 89.8 & 90.9 & 90.9 & 90.5 & 90.1 \\
\hline 26 & 482 & 781 & 13.4 & $1.003 \times 10^{-3}$ & $1.012 \times 10^{-4}$ & 125.5 & 125.6, & 125.8 & 125.6 & 129.5 \\
\hline
\end{tabular}

Table 1. Results for variation of experimental parameters in equation (1) 


\section{Investigation of Validity of Basic Equation}

A variety of experimental parameters were selected covering a range of values of $Q, V_{\mathrm{i}}, V, C_{\mathrm{o}}^{\varsigma}$ and $C_{\mathrm{o}}^{\mathrm{R}}$ as shown in Table 1 . Twenty-six different combinations were used and, for each, injections were performed in triplicate. The over-all agreement between the experimental and the calculated values of $\Delta t_{\mathrm{eq}}$ was assessed together with the relationship observed between $\Delta t_{\mathrm{eq}}$ and each of the parameters $Q, V_{\mathrm{i}}, V$ and $C_{\mathrm{o}}^{\mathrm{s}}$. The volumes of the injection loops and the mixing chambers used were calculated from the mass of mercury that the various components would contain. The precision of this procedure was established by making ten replicate measurements of the loop nominally labelled by the suppliers as $500 \mu \mathrm{l}$. Flow-rates were measured by collecting and weighing the effluent from the detector over the time period approximately required for the three injections, as measured by a quartz digital stopwatch. The conversion from mass to volume was made assuming a specific gravity of unity. The detector wavelength was $280 \mathrm{~nm}$ throughout.

\section{Extending the Calibration Range}

Solutions covering the range $5 \times 10^{-6-5} \mathrm{M} \mathrm{NaOH}$ were prepared by serial dilution of the $5 \mathrm{~m}$ stock solution, and $498 \mu \mathrm{l}$ of each was injected into the acidified indicator carrier stream. The gradient tube was used as the mixing device and the detector was tuned to $620 \mathrm{~nm}$. A graph of $\Delta t_{\mathrm{eq}}$ versus $\ln C_{\mathrm{o}}^{\delta}$ was constructed and evaluated.

\section{Results and Discussion}

\section{Preliminary Experiments}

It was found that whereas all gradient-forming devices gave calibration graphs with correlation coefficients greater than 0.99 , there was an improvement in linearity in progressing from tubes to the gradient tube to the mixing chamber. Straight tubes were found to be the poorest and an improvement could be achieved by coiling the tube. This also had the effect of reducing the slope of the calibration, i.e., apparently reducing the volume of the equivalent mixing chamber. There was no obvious relationship between the volume of this mixing chamber and the real volume of the coil or a combination of the coil and the injection loop, although this has been reported for short lengths of tubing and low injection volumes.? Although the best linearity was obtained with the mixing chamber, the sampling frequency was the lowest of the various devices investigated owing to the relatively long time required to wash out the chamber. Hence manifolds containing real mixing chambers were selected for studies on the validity of equation (1) whereas a manifold containing the gradient tube

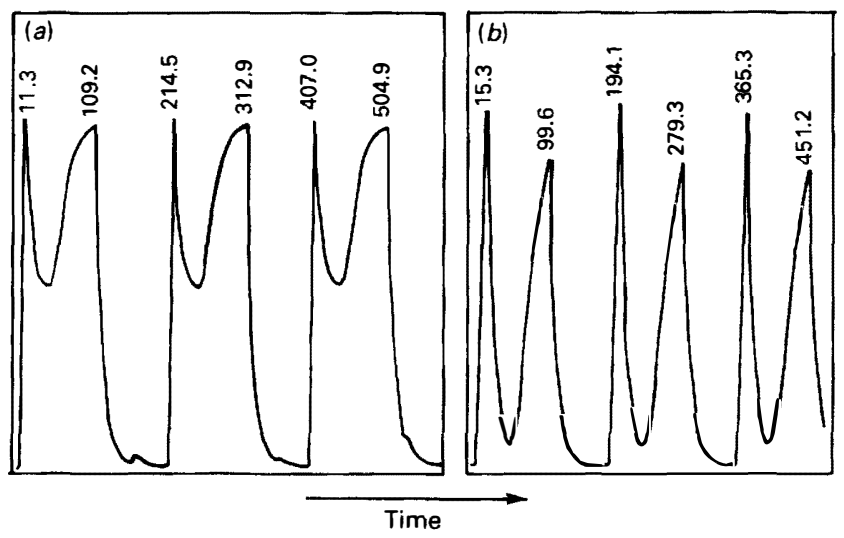

Fig. 2. Typical doublet peak output from the computing integrator. (a) Results of run 4 and (b) results of run 12 (see Table 1). The integrator gives the retention times to the nearest $0.1 \mathrm{~s}$ was used for a practical example as a compromise between the linearity of the calibration and the sampling frequency.

\section{Validity of Equation (1)}

The results obtained are shown in Table 1 with some typical peak shapes given in Fig. 2. The line of regression of the mean of the measured $\Delta t_{\mathrm{eq}}$ values on the calculated $\Delta t_{\mathrm{eq}}$ values, calculated by the method of least squares, had a slope of 0.969 \pm 0.018 , an intercept of $0.730 \pm 1.77$ and a correlation coefficient of 0.9990 . The uncertainty values quoted for the slope and intercept are the $95 \%$ confidence intervals. This analysis of the results assumes that there is no uncertainty in the calculated values of $\Delta t_{\text {eq }}$. However, this is not so as all the terms involved are subject to experimental error. The standard deviation for the operation of determining the volume of the $500-\mu \mathrm{l}$ loop was $0.022 \mathrm{~g}$ for a mean mass of mercury of $6.528 \mathrm{~g}$. This corresponds to a mean volume of 482 $\pm 1 \mu \mathrm{l}$ where the uncertainty quoted is the $95 \%$ confidence interval. The values for the flow-rates are averaged over the time taken to obtain the three replicate measurements, which amounted to several minutes in some instances. The peristaltic pump was kept at the same nominal setting for runs 8-22 and there is probably a decrease in the flow-rate during this period of several hours as the pump tubing becomes distorted.

The results for the variation of $\Delta t_{\mathrm{eq}}$ with $C_{\mathrm{o}}^{\mathrm{S}}$ were taken from runs 1-4 in Table 1. A least-squares analysis of the line of regression of $\Delta t_{\mathrm{eq}}$ on $\ln C_{\mathrm{o}}^{\mathrm{s}}$ had a slope of $11.52 \pm 1.46$, an intercept of $151.0 \pm 12.3$ and a correlation coefficient of 0.9991 . The calculated values of the slope and the intercept are 12.1 and 156.3 , respectively.

The variation of $\Delta t_{\mathrm{eq}}$ with the volume injected, $V_{\mathrm{i}}$, was analysed for runs $8-12$ in Table 1 . The results of the least-squares analysis of the line of regression of $\Delta t_{\mathrm{eq}}$ on $\ln \left[\exp \left(V_{\mathrm{i}} / V\right)-1\right]$ was a slope of $14.14 \pm 0.50$ and an intercept of $32.08 \pm 1.03$. The calculated values are 13.85 and 32.00 , respectively.

The variation of $\Delta t_{\mathrm{eq}}$ with the mixing chamber volume, $V$, was analysed for runs $11,14,18$ and 22 in Table 1 . The results of the least-squares fit of the data to the line of regression $\Delta t_{\text {eq }}$ on $(V / Q) \ln \left[\left(\exp \left(V_{\mathrm{i}} / V\right)-1\right] C_{\mathrm{o}}^{\mathrm{S}} / C_{\mathrm{o}}^{\mathrm{R}}\right.$ was a slope of $0.93 \pm 0.12$, an intercept of $4.69 \pm 12.1$ and a correlation coefficient of 0.9992 . The calculated values of the slope and the intercept are 1 and 0 , respectively. At first sight the effect of changing $V$ observed here is the opposite of what might be expected intuitively, namely that increasing the mixing chamber volume should decrease the peak separation. Rearranging equation (4) as

$$
\Delta t_{\mathrm{eq}}=(V / Q) \ln C_{\mathrm{o}}^{\mathrm{s}} / C_{\mathrm{o}}^{\mathrm{R}}+(V / Q) \ln \left[\exp \left(V_{\mathrm{i}} / V\right)-1\right] \ldots
$$

shows the reason for the increase in $\Delta t_{\mathrm{eq}}$ with $V$ (at least up to the maximum value) as the first term on the right-hand side of equation (7) increases linearly with $V$ at a rate that is faster than the decrease in value of the second term on the right-hand side. This is readily shown by plotting the variation in $\Delta t_{\text {eq }}$ with $V$ for each of these two terms. Differentiating equation (7) and setting the resulting equation equal to zero, to give the condition for a turning point in the function, gives $\exp \left(V_{\mathrm{i}} / V\right)=\left(V / V_{\mathrm{i}}\right)\left[\exp \left(V_{\mathrm{i}} / V\right)-1\right] \times$

$$
\ln \left[\exp \left(V_{\mathrm{i}} / V\right)-1\right] C_{\mathrm{o}}^{\mathrm{s}} / C_{\mathrm{o}}^{\mathrm{R}}
$$

This equation can be solved readily by the method of Newton's approximation and for the values of the experimental parameters used here predicts that $\Delta t_{\mathrm{eq}}$ will have a maximum value of $149.4 \mathrm{~s}$ for a mixing tank volume of $3318 \mu \mathrm{l}$. These values are confirmed by calculations based on substitution in equation (4). The relationship between the maximum value of $\Delta t_{\mathrm{eq}}\left[\Delta t_{\mathrm{eq}(\mathrm{m})}\right]$ and the corresponding dispersion coefficient value, $D_{(\mathrm{m})}$, is given by

$$
\Delta t_{\mathrm{eq}(\mathrm{m})}=D_{(\mathrm{m})} V_{\mathrm{i}} / Q
$$


The appearance of a maximum in the $\Delta t_{\mathrm{eq}}$ versus $V$ relationship cannot be exploited for quantitative analytical purposes as the value of $V$, giving the maximum value, varies with the concentration injected. However, if $C^{\mathrm{S}} / C^{\mathrm{R}}$ is always greater than zero, then selection of the smallest value of $V$ will not necessarily give the largest value of $\Delta t_{\mathrm{eq}}$. There may, of course, be other factors to be considered in the choice of experimental parameters, such as sampling frequency.

Finally the variation of $\Delta t_{\mathrm{eq}}$ with flow-rate, $Q$, was analysed based on the individual results (rather than the mean $\Delta t_{\mathrm{eq}}$ values) for runs $15,23-26$ in Table 1 . The regression analysis of $\Delta t_{\mathrm{eq}}$ on $\mathrm{l} / Q$ gave a slope of $1720 \pm 35$, an intercept of -2.63 \pm 1.73 and a correlation coefficient of 0.9994 . The calculated value of the slope was 1674 and that of the intercept was 0 .

All the uncertainties quoted are $95 \%$ confidence intervals, and for the variation of $\Delta t_{\mathrm{eq}}$ with most of the experimental parameters being considered individually, there is no significant difference between the values of the slopes and the intercepts found from the least-squares analysis of the data and these values calculated on the basis of equation (1). However, to some extent this is a function of the small number of data points taken. When the number of data points is increased, as in the variation of $\Delta t_{\mathrm{eq}}$ with flow-rate in which 15 data points were available, significant differences do become apparent. This also applies to the results taken overall where the slope of the regression line indicates that the calculated values of $\Delta t_{\text {eq }}$ are significantly greater than the measured values. Despite the limitations of the statistical method used to reach this conclusion, there is a good reason why this difference should be observed, namely the method of injection. The model assumes that time injection is used but the experimental arrangement used here, in common with most other flow injection systems described in the literature, employed slug injection. The rear of the injected slug therefore undergoes a greater dispersion than the leading edge as discussed elsewhere ${ }^{14}$ and the system behaves as if the value of $V$, the mixing chamber volume, is larger than it actually is.

\section{Extending the Calibration Range}

The results obtained are shown in Table 2. A linear regression analysis by least squares of the graph of $\Delta t_{\mathrm{eq}}$ versus $\log C_{\mathrm{o}}^{\mathrm{s}}$ (excluding the point corresponding to $5 \times 10^{-6} \mathrm{M}$ ) gave a slope of $9.65 \pm 0.33$, an intercept of $104.5 \pm 0.8$ and a correlation coefficient of 0.9997 . Hence, as far as obtaining linear calibration for making quantitative analytical applications is concerned it is unnecessary to have a real mixing chamber in the manifold. Much smaller gradient-forming devices with a consequent increase in sampling rate can be used. The ease with which concentrations of $\mathrm{OH}^{-}$as high as $5 \mathrm{~m}$ can be measured by a system such as this suggests that the doublet peak flow injection technique could be a useful alternative in situations where the response of a glass electrode can be

Table 2. Results for calibration for $\mathrm{OH}^{-}$using gradient tube manifold

$\begin{array}{crrr}C_{\mathrm{o}}^{\mathrm{S}} / \mathrm{M} & \operatorname{Ln} C_{\mathrm{o}}^{\mathrm{s}} & \log C_{\mathrm{o}}^{\mathrm{s}} & \Delta t_{\mathrm{eq}} / \mathrm{s} \\ 5.0 & 1.609 & 0.699 & 164.4 \\ 0.50 & -0.693 & -0.301 & 130.2 \\ 5.0 \times 10^{-2} & -2.996 & -1.301 & 117.0 \\ 5.0 \times 10^{-3} & -5.298 & -2.301 & 99.6 \\ 5.0 \times 10^{-4} & -7.601 & -3.301 & 79.2 \\ 5.0 \times 10^{-5} & -9.903 & -4.301 & 68.4 \\ 5.0 \times 10^{-6} & -12.206 & -5.301 & 70.2\end{array}$

inaccurate owing to the interference from the high concentration of cation(s). The system no doubt could be used in the alternative configuration with a dilute alkali carrier stream for measuring high concentrations of $\mathrm{H}^{+}$.

\section{Conclusions}

The well stirred mixing chamber model produces an equation relating the time interval between the doublet peaks, produced by monitoring the product profile during a flow injection titration, to the experimental variables in good agreement with the experimentally determined relationship. The technique has considerable potential as a quantitative method as it enables the working range of a spectrophotometric method (and others no doubt) to be extended over several orders of magnitude of concentration. The equations derived also readily allow the conditions necessary for a doublet peak to be produced to be calculated and hence the lower end of the working range is not necessarily sacrificed in order to achieve the improvement at the upper end. Of course, at sample concentrations too low to obtain a doublet peak from a particular manifold, peak height can be used as a quantitative parameter in the normal way.

It is difficult to resist a comparison of the equation for the doublet peak method with the Nernst equation. Adopting the convention that the value of $\Delta t_{\mathrm{eq}}$ obtained for the reactants in their standard states is designated $\Delta t_{\text {eq }}^{\circ}$, equation (1) can be rewritten as

$$
\Delta t_{\mathrm{eq}}=\Delta t_{\mathrm{eq}}^{\mathrm{o}}+(2.303 \mathrm{~V} / Q) \log \left(C_{\mathrm{o}}^{\mathrm{S}} / C_{\mathrm{o}}^{\mathrm{R}}\right)
$$

This is for a 1:1 reaction. More general versions of equation (10) can be readily derived from the equations given in the appendices of reference 13. Given that devices based on the Nernst equation find wide application, viewing the quantitative basis of the doublet peak method in a similar fashion can open up some interesting applications for the technique.

Financial support from the SERC for the purchase of equipment is gratefully acknowledged.

\section{References}

1. Wolf, W. R., and Stewart, K. K., Anal. Chem., 1979, 51, 1201.

2. Růžička, J., Hansen, E. H., and Mosbaek, H., Anal. Chim. Acta, 1977, 92, 235.

3. Blaedel, W. R., and Lessing, R. H., Anal. Chem., 1964, 36, 1617

4. Fleet, B., and Ho, A. Y. W., Anal. Chem., 1974, 46, 9.

5. Pungor, E., Toth, K., and Nagy, G., Anal. Chem., 1975, 47, 1460 .

6. Pardue, H. L., and Fields, B., Anal. Chim. Acta, 1981, $124,39$.

7. Ramsing, A. U., Růžička, J., and Hansen, E. H., Anal. Chim. Acta $, 1981,129,1$.

8. Rhee, J.-S., and Dasgupta, P. K., Mikrochim. Acta, 1985, 3, 49

9. Rhee, J.-S., and Dasgupta, P. K., Mikrochim. Acta, 1985, 3, 107.

10. Stewart, K. K., and Rosenfeld, A. G., Anal. Chem., 1982, 54, 2368.

11. Tyson, J. F., Analyst, 1984, 109, 319.

12. Rủžička, J., and Hansen, E. H., "Flow Injection Analysis," Wiley, New York, 1981, pp. 137, 138 and Fig. 6.6.

13. Tyson, J. F., Anal. Chim. Acta, 1986, 179, 131.

14. Stone, D. C., and Tyson, J. F., Analyst, 1987, 112, 515

Paper $A 6 / 353$ Received September 17th, 1986 Accepted November 19th, 1986 